\title{
On the Functionally Countable Subalgebra of $C(X)$
}

\author{
M. Ghadermazi (*) - O. A. S. KaramZadeh $(* *)$ - M. Namdari $(* *)$
}

Abstract - Let $C_{c}(X)=\{f \in C(X): f(X)$ is countable $\}$. Similar to $C(X)$ it is observed that the sum of any collection of semiprime (resp. prime) ideals in the ring $C_{c}(X)$ is either $C_{c}(X)$ or a semiprime (resp. prime) ideal in $C_{c}(X)$. For an ideal $I$ in $C_{c}(X)$, it is observed that $I$ and $\sqrt{I}$ have the same largest $z_{c}$-ideal. If $X$ is any topological space, we show that there is a zero-dimensional space $Y$ such that $C_{c}(X) \cong C_{c}(Y)$. Consequently, if $X$ has only countable number of components, then $C_{c}(X) \cong C(Y)$ for some zero-dimensional space $Y$. Spaces $\mathrm{X}$ for which $C_{c}(X)$ is regular (called $C P$-spaces) are characterized both algebraically and topologically and it is shown that $P$-spaces and $C P$-spaces coincide when $X$ is zerodimensional. In contrast to $C^{*}(X)$, we observe that $C_{c}(X)$ enjoys the algebraic properties of regularity, $\aleph_{0}$-selfinjectivity and some others, whenever $C(X)$ has these properties. Finally an example of a space $X$ such that $C_{c}(X)$ is not isomorphic to any $C(Y)$ is given.

Mathematics Subject Classification (2010). Primary 54C30, 54C40; 54C05; 54G12; Secondary 13C11, 16H20.

KEYwords. Functionally countable subring, socle, $z_{c}$-idea, regular ring, $P$-space, $C P$-space, Scattered space, $\aleph_{0}$-selfinjective, zero-dimensional space, component.

\section{Introduction}

Unless otherwise mentioned all topological spaces $X$ are infinite completely regular Hausdorff and we will employ the definitions and notation used in [9]. A celebrated result due to Rudin [23], Pelczynski and Sema-

(*) Indirizzo dell'A.: Department of Mathematics, University of Kurdistan, P.O. Box 416, Sanandaj, Iran

E-mail: mostafa.ghadermazi@uok.ac.ir,

(**) Indirizzo degli A.: Department of Mathematics, Shahid Chamran University, Ahvaz, Iran

E-mail: karamzadeh@ipm.ir_namdari@ipm.ir 
deni [21], states that if $X$ is a compact space then $X$ is functionally countable (i.e., each member of $C(X)$ has countable image) if and only if $X$ is scattered (i.e., every nonempty subspace of $X$ contains an isolated point) see also [19, Proposition 3.1] and [18]. Recently the if part of this interesting result has been generalized to compact a-scattered spaces in [8]. One trivially observes that the subset of $C(X)$, where $X$ is any space not necessarily compact, consisting of those members with countable image is a subring of $C(X)$ containing the real numbers, i.e., an $\mathbb{R}$-subalgebra of $C(X)$, and we denote it by $C_{c}(X)$. Motivated by the first result mentioned above and the latter trivial fact we are led to study $C_{c}(X)$ and will try to connect its algebraic properties to topological properties of $X$. It turns out $C_{c}(X)$, although not isomorphic to any $C(Y)$ in general, enjoys most of the important properties of $C(X)$ (note, some of these properties do not hold for $C^{*}(X)$ unless $X$ is finite). It seems up to now the only well-known subring of $C(X)$ which, parallel to $C(X)$ has played a major role in the context of $C(X)$, is $C^{*}(X)$. But we should notice that $C^{*}(X)$ is in fact $C(Y)$, where $Y=\beta X$, that is to say $C(X)$ and $C^{*}(X)$ are of the same type and hence in this sense the description of a proper subring of $C(X)$ fits $C_{c}(X)$ to a tee. Let us, without further ado, give an outline of this paper. After presenting some preliminary results of $C(X)$ which are trivially shared by $C_{c}(X)$ in Section 2 , we introduce the $z_{c}$-ideals in Section 3 , and prove the fact that for any ideal $I$ in $C_{c}(X), I$ and $\sqrt{I}$ have the same largest $z_{c}$-ideal. It is also observed that the sum of any family of semiprime (resp. prime) ideals in $C_{c}(X)$ is either $C_{c}(X)$ or a semiprime (resp. prime) ideal in $C_{c}(X)$. In Section 4 , topological spaces in which points and closed sets are separated by elements in $C_{c}(X)$ are called c-completely regular space and it is observed that these spaces coincide with zero-dimensional ones. Consequently for any topological space $X$ we prove that there exists a zero-dimensional space $Y$ which is a continuous image of $X$ and $C_{c}(X) \cong C_{c}(Y)$. Section 5, is devoted to the introduction of $C P$-spaces (i.e., spaces for which $C_{c}(X)$ is a regular ring). It is observed that every $P$-space is a $C P$-space and a characterization of $C P$ spaces similar to the one for $P$-spaces is given (see $[9,4 \mathrm{~J}]$ ). In particular, we show that $X$ is a $C P$-space if and only if whenever $\left\{f_{n}\right\}_{n=1}^{\infty} \subseteq C_{c}(X)$ then $\bigcap_{n=1}^{\infty} Z\left(f_{n}\right)$ is an open zero-set of the form $Z(f)$, where $f \in C_{c}(X)$. Consequently in zero-dimensional spaces, $P$-spaces and $C P$-spaces are the same. In Section 6, we show that some important algebraic properties of $C(X)$ such as regularity, $\aleph_{0}$-selfinjectivity and some others are shared by $C_{c}(X)$ but not by $C^{*}(X)$. Finally in Section 7 , we present some examples to show that $C_{c}(X)$ may not be isomorphic to $C(Y)$ for any topological space $Y$. 


\section{Preliminaries}

In this section we aim to state, without proofs, some of the elementary properties of $C_{c}(X)$ which are needed in the subsequent sections (note, the proofs are more or less similar to the corresponding ones for $C(X)$ ). It is evident that whenever $X$ is connected then $C_{c}(X)=\mathbb{R}$, otherwise $\mathbb{R} f C_{c}(X)$. It is also worth mentioning that if $X$ is either equal to $\beta \mathbb{N}$ or to $\beta \mathrm{Q}$, then $C_{c}(X)$ is a proper $\mathbb{R}$-subalgebra of $C(X)$ which is not isomorphic to $\mathbb{R}$ or a direct product of copies of $\mathbb{R}$ (note, $X$ is strongly zero-dimensional, see [6, Theorems 6.2.7, 6.2.12], but it is neither a scattered space nor a $P$-space, see the first few lines of the introduction and our Corollary 5.7). We also note that $f \vee g=\frac{f+g+|f-g|}{2} \in C_{c}(X)$ for all $f, g \in C_{c}(X)$, hence $C_{c}(X)$ is a sublattice of $C(X)$. For $f \in C_{c}(X)$ and $f>0$ we note that there exists $k \in C_{c}(X)$ with $f=k^{2}$. We also note that whenever $f \in C_{c}(X)$ and $f^{r}$ is defined with $f^{r} \in C(X)$, where $r \in \mathbb{R}$, then $f^{r} \in C_{c}(X)$. Let us put $C_{c}^{*}(X)=C_{c}(X) \cap C^{*}(X)$, then every homomorphism $\varphi: C_{c}(X) \rightarrow C_{c}(Y)$ takes $C_{c}^{*}(X)$ into $C_{c}^{*}(Y)$. It is clear that if $X$ is pseudocompact then $C_{c}^{*}(X)=C_{c}(X)$. But we may have $C_{c}^{*}(X)=C_{c}(X)$ without the pseudocompactness of $X$, for consider $X=(-1,0) \cup\{1,2, \ldots, n\}$ as the subspace of $\mathbb{R}$, then $C_{c}^{*}(X)=C_{c}(X)$.

We recall that the socle of $C(X)$, denoted by $C_{F}(X)$, which is the sum of all minimal ideals of $C(X)$, consists of all functions in $C(X)$ which vanish everywhere except on finite number of points of $X$, see [16, Proposition 3.3]. Clearly $C_{F}(X)$ is an ideal in both $C(X)$ and $C_{c}(X)$. Motivated by this we give the next definition.

Definition 2.1. For a topological space $X$ we put

$$
C^{F}(X)=\{f \in C(X):|f(X)|<\infty\} .
$$

It is manifest that $C^{F}(X)$ is a subring of $C_{c}(X)$ and

$$
C_{F}(X) \subseteq C^{F}(X) \subseteq C_{c}(X) \subseteq C(X) .
$$

It goes without saying that if $C(X, K)$ is a subring of $C(X)$ such that each $f \in C(X, K)$ takes values in a countable subring $K$ of $\mathbb{R}$, then it is a subring of $C_{c}(X)$. Let us for the sake of brevity put $C(X, Z)=C_{i}(X)$, $C(X, Q)=C_{r}(X)$ and note that these subrings are unfortunately not $\mathbb{R}$ subalgebras of $C(X)$. But in this article we are mainly interested in $C_{c}(X)$ 
and $C^{F}(X)$ and note that the latter two subrings of $C(X)$ are $\mathbb{R}$-subalgebras of $C(X)$ containing the socle of $C(X)$.

Remark 2.2. Let $X$ be a space with $C(X)=C_{c}(X)$ and $Y$ be a pseudocompact space and $Z=X \cup Y$ be the free sum of $X, Y$. Then $C(Z)=C_{c}(Z)+C^{*}(Z)$.

REMARK 2.3. Let $f \in C_{c}(X)$, then $f$ is a unit in $C_{c}(X)$ if and only if $Z(f)=\varnothing$, a fact which is not true for $C^{*}(X)$ in general.

We recall that $[9,1 \mathrm{D}(1)]$ plays a useful role in the context of $C(X)$. The following is the counterpart for $C_{c}(X)$.

LEMma 2.4. If $f, g \in C_{c}(X)$ and $Z(f)$ is a neighborhood of $Z(g)$, then $f=$ gh for some $h \in C_{c}(X)$.

Corollary 2.5. If $f, g \in C_{c}(X)$, and $|f| \leq|g|^{r}, r>1$, then $f=g h$ for some $h \in C_{c}(X)$. In particular, if $|f| \leq|g|$, then whenever $f^{r}$ is defined for $r>1, f^{r}$ is a multiple of $g$.

Convention. Let us put $Z_{c}(X)=\left\{Z(f): f \in C_{c}(X)\right\}$, where $X$ is a topological space.

Remark 2.6. Lemma 2.4, Corollary 2.5 are also valid in $C^{F}(X)$ and $C_{r}(X)$ but not in $C_{i}(X)$.

Definition 2.7. Two subsets $A$ and $B$ of a topological space $X$ are said to be countably separated ( $c$-separated) in $X$ if there is an element $f \in C_{c}(X)$ such that $f(A)=1, f(B)=0$.

The next result is the counterpart of [9, Theorem 1.15].

Theorem 2.8. Two subsets $A, B$ of a space $X$ are c-separated if and only if they are contained in disjoint members of $Z_{c}(X)$. Moreover, $c$-separated sets have disjoint zero-set neighborhoods in $Z_{c}(X)$.

COROLlary 2.9. If $A, A^{\prime}$ are c-separated in $X$, then there are zero-sets $F, Z$ in $Z_{c}(X)$ with $A \subseteq X \backslash Z \subseteq F \subseteq X \backslash A^{\prime}$.

Similar to a $z$-filter, one can define a $z_{c}$-filter on $X$. 
Definition 2.10. $\varnothing \neq F \subseteq Z_{c}(X)$ is called a $z_{c}$-filter on $X$ if $F$ satisfies the following conditions.

(i) $\varnothing \notin F$.

(ii) $Z_{1}, Z_{2} \in F$, then $Z_{1} \cap Z_{2} \in F$.

(iii) $Z \in F, Z^{\prime} \in Z_{c}(X)$ with $Z^{\prime} \supseteq Z$, then $Z^{\prime} \in F$.

Clearly, for every ideal $I$ in $C_{c}(X), Z_{c}[I]=\{Z(f): f \in I\}$ is a $Z_{c}$-filter on $X$ and conversely, if $F$ is a $Z_{c}$-filter on $X$, then $Z^{-1}[F]=$ $\left\{f \in C_{c}(X): Z(f) \in F\right\}$ is an ideal in $C_{c}(X)$. Moreover, every $z_{c}$-filter $F$ is of the form $F=Z_{c}[I]$ for some ideal $I$ in $C_{c}(X)$ and for any ideal $A$ in $C_{c}(X), Z^{-1}\left[Z_{c}[A]\right]$ is an ideal in $C_{c}(X)$ containing $A$.

Definition 2.11. An ideal $I$ in $C_{c}(X)$ is called a $z_{c}$-ideal if whenever $Z(f) \in Z_{c}[I], f \in C_{c}(X)$, then $f \in I$.

REMARK 2.12. Clearly, every $z_{c}$-ideal in $C_{c}(X)$ is an intersection of prime ideals in $C_{c}(X)$. Prime $z_{c}$-filters and $z_{c}$-ultrafilters are defined similar to their counterparts in [9] and one can easily see that in fact all the results in [9, Chapter 2] are trivially valid if we replace $C(X)$ by $C_{c}(X)$.

We need the following results too.

Theorem 2.13. Let $Q$ be any $z_{c}$-ideal in $C_{c}(X)$. Then the following statements are equivalent.

1. $Q$ is a prime ideal in $C_{c}(X)$.

2. $Q$ contains a prime ideal in $C_{c}(X)$.

3. For all $f, g \in C_{c}(X)$ with $f g=0$, we have either $f \in Q$ or $g \in Q$.

4. For each $f \in C_{c}(X)$, there exists a zero-set in $Z_{c}[Q]$ on which $f$ does not change sign.

COROLlary 2.14. Every prime ideal in $C_{c}(X)$ is contained in a unique maximal ideal in $C_{c}(X)$.

REMark 2.15. Let us define an ideal $I$ in $C^{F}(X)$ to be a $z_{F}$-ideal if $Z(f) \in Z_{F}[I]=\{Z(g): g \in I\}, f \in C^{F}(X)$, then $f \in I$. Now it is easy to see that the previous two results are also valid in $C^{F}(X)$ if we replace $z_{c}$-ideal by $z_{F}$-ideal and $Z_{c}[Q]$ by $Z_{F}[Q]$ in these results. It is manifest that these results are valid for some more applicable subrings, such as $C_{r}(X), C_{i}(X)$, of $C_{c}(X)$. 


\section{3. $z_{c}$-ideals}

If $x \in X$ and $M_{x}^{c}=M_{x} \cap C_{c}(X)$, where $M_{x}$ consists of those elements of $C(X)$ which vanish at $x$, then $M_{x}^{c}$ is a maximal ideal in $C_{c}(X)$, for consider $\varphi: C_{c}(X) \stackrel{\text { onto }}{\longrightarrow} \mathbb{R}$, where $\varphi(f)=f(x)$, hence $\operatorname{ker} \varphi=M_{x}^{c}$ and we are done. Consequently the Jacobson radical of $C_{c}(X)$ is zero, i.e., $J\left(C_{c}(X)\right)=0$. It is well-known that the sum of two $z$-ideals in $C(X)$ is either $C(X)$ or a $z$-ideal, see [9, Lemma 14.8]. The usual proof of this result depends on the properties of $\beta X$ which are inapplicable to $C_{c}(X)$. But fortunately the proof of this result in [24] can be modified for $C_{c}(X)$ and is presented below.

Lemma 3.1. Let $f, g, l \in C_{c}(X), Z(f) \supseteq Z(g) \cap Z(l)$ and define

$$
h(x)=\left\{\begin{array}{ll}
0, & x \in Z(g) \cap Z(l) \\
\frac{f g^{2}}{g^{2}+l^{2}}, & x \notin Z(g) \cap Z(l)
\end{array}, \quad k(x)=\left\{\begin{array}{ll}
0, & x \in Z(g) \cap Z(l) \\
\frac{f l^{2}}{g^{2}+l^{2}}, & x \notin Z(g) \cap Z(l)
\end{array} .\right.\right.
$$

Then we have the following conditions.

1. $|k| \vee|h| \leq|f|$.

2. $f=h+k$.

3. $f l^{2}=k\left(g^{2}+l^{2}\right), f g^{2}=h\left(g^{2}+l^{2}\right)$.

4. $h, k \in C_{c}(X)$.

Proof. It suffices to show that $h, k$ are continuous. By similarity, we may only show that $h$ is continuous. To this end, we prove that $h$ is continuous at any point $x \in Z(g) \cap Z(l)$ and this completes the proof. Since $f(x)=0$, we infer that for any $\varepsilon>0$ there exists a neighborhood $U$ of $x$ with $x \in U \subseteq f^{-1}(-\varepsilon, \varepsilon)$. Now by (1) and the fact that $f(U) \subseteq(-\varepsilon, \varepsilon)$ we have $h(U) \subseteq(-\varepsilon, \varepsilon)$, i.e., $h$ is continuous.

Corollary 3.2. Let $A, B$ be two $z_{c}$-ideals in $C_{c}(X)$. Then either $A+B=C_{c}(X)$ or $A+B$ is a $z_{c}$-ideal.

Proof. Let $A+B \neq C_{c}(X)$ and $f \in C_{c}(X)$ be an element with $Z(f)=Z\left(f^{\prime}\right)$, where $f^{\prime} \in A+B$. We are to show that $f \in A+B$. But $f^{\prime}=g+l$, where $g \in A, l \in B$. Clearly, $Z(f)=Z\left(f^{\prime}\right) \supseteq Z(g) \cap Z(l)$. Now let $h, k$ be as in the previous lemma, hence $f=h+k$. But $Z(h) \supseteq Z(g)$ and $Z(k) \supseteq Z(l)$ and since $A, B$ are $z_{c}$-ideals we infer that $h \in A, k \in B$. Hence $f \in A+B$ and we are through. 
Corollary 3.3. Let $F=\left\{A_{i}\right\}_{i \in I}$ be a collection of $z_{c}$-ideals in $C_{c}(X)$. Then either $\sum_{i \in I} A_{i}=C_{c}(X)$ or $\sum_{i \in I} A_{i}$ is a $z_{c}$-ideal.

Using the fact that each minimal prime ideal in a subring of a ring $R$ is the contraction of a minimal prime ideal of $R$ and the fact that minimal prime ideals in $C(X)$ are $z$-ideals, we immediately have the following corollary.

Corollary 3.4. Every minimal prime ideal in $C_{c}(X)$ is a $z_{c}$-ideal.

The next result is the consequence of the previous two corollaries and Theorem 2.13 .

Corollary 3.5. Let $F=\left\{P_{i}\right\}_{i \in I}$ be a collection of minimal prime ideals in $C_{c}(X)$. Then either $\sum_{i \in I} P_{i}=C_{c}(X)$ or $P=\sum_{i \in I} P_{i}$ is a prime ideal in
$C_{c}(X)$.

Let us recall that a subring $A$ of $C(X)$ is called absolutely convex if $f \in C(X), g \in A$, and $|f| \leq|g|$ imply that $f \in A$. In [24], certain properties of $C(X)$ are extended, in an easy way, to absolutely convex subrings of $C(X)$. Although, $C_{c}(X)$ may not be absolutely convex in general, but if we borrow the proofs of [24, Lemma 2.1, Lemma 5.1, Theorem 5.2, Theorem 5.3], word-for-word, we obtain the same results for $C_{c}(X)$ in the following sequence of corollaries, see also [17, Corollary $2.3]$ and [9, 14B.1].

Corollary 3.6. A prime ideal $P$ in $C_{c}(X)$ is absolutely convex.

Corollary 3.7. The sum of a collection of semiprime ideals in $C_{c}(X)$ is a semiprime ideal or is the entire ring $C_{c}(X)$.

Corollary 3.8. Let $P$ be a prime ideal in $C_{c}(X)$. Then the ring $C_{c}(X) / P$ is totally ordered and its prime ideals are comparable.

The next corollary is much stronger than Corollary 3.5.

Corollary 3.9. Let $\left\{P_{i}\right\}_{i \in I}$ be a collection of semiprime ideals in $C_{c}(X)$ such that at least one of $P_{i}$ 's is a prime ideal, then $\sum_{i \in I} P_{i}$ is a prime ideal (or all of $C_{c}(X)$ ). 
In [20, Proposition 2.4] and [2, Proposition 3.2] and [4, Corollary 2.3] it is shown that for any ideal $I$ in $C(X), I$ and $\sqrt{I}$ have the same largest $z$-ideal. The following is the counterpart for $C_{c}(X)$. But before presenting the result, we note that whenever $f \in C_{c}(X)$ and $g=\sum_{n=1}^{\infty}|f|^{r_{n}} \in C(X)$, where $r_{n}$ is a positive real number, then $g \in C_{c}(X)$ (note, let $f(X)=\left\{a_{n}\right\}_{n=1}^{\infty}$, then $g(X)=\left\{b_{i}\right\}_{i=1}^{\infty}$, where $\left.b_{i}=\sum_{n=1}^{\infty}\left|a_{i}\right|^{r_{n}}\right)$.

THEOREM 3.10. Let I be an ideal in $C_{c}(X)$. Then I and $\sqrt{I}$ have the same largest $z_{c}$-ideal.

PRoof. We first observe that the sum of all $z_{c}$-ideals in a proper ideal is a proper $z_{c}$-ideal, by Corollary 3.3. Hence it suffices to show that if $A$ is a $z_{c}$-ideal in $\sqrt{I}$, then $A \subseteq I$. To see this, let $f \in A$ and by replacing $f$ by $\frac{f}{1+f^{2}} \in A$, we may assume that $|f| \leq 1$. Now define $g=\sum_{n=1}^{\infty} \frac{|f|^{\frac{1}{n}}}{2^{n}}$, hence $g \in C_{c}(X)$, by the above comment. Since $Z(g)=Z(f)$ and $f \in A$ we infer that $g \in A \subseteq \sqrt{I}$. Thus $g^{n} \in I$ for some $n>1$. But $2^{-n^{2}}|f|^{\frac{1}{n^{2}}} \leq g$, i.e., $|f| \leq\left(2^{n^{3}} g^{n}\right)^{n}$, which implies that $g^{n} \mid f$, by Corollary 2.5, therefore $f \in I$ and we are done.

REMARK 3.11. If we replace $C_{c}(X)$ by $C^{F}(X)$ and $z_{c}$-ideals by $z_{F}$-ideals, then all the results of this section remain valid for $C^{F}(X)$. But, we should emphasize that the function $g$ defined in the previous proof may not be in some of the natural subrings of $C_{c}(X)$, for example $g$ is not in $C_{r}(X)$, hence the previous proof cannot be extended to other subrings of $C_{c}(X)$ in general. But we should also emphasize that all the results in this section except Theorem 3.10, remain valid (with the same proofs) for $C_{r}(X)$ and more generally for $C(X, K)$, where $K$ is a subfield of $\mathbb{R}$. Using the comment preceding Corollary 3.4, the counterpart of Corollary 3.4, can be easily proved for $C_{i}(X)$, but the other results of this section may not be true for the latter ring.

\section{Zero-dimensional spaces and $C_{c}(X)$}

We begin with the following definition.

Definition 4.1. A Hausdorff space $X$ is called countably completely regular (briefly, $c$-completely regular) if whenever $F \subseteq X$ is a closed set and $x \notin F$, then there exists $f \in C_{c}(X)$ with $f(F)=0$ and $f(x)=1$. 
Clearly, a Hausdorff space $X$ is $c$-completely regular if and only if whenever $F \subseteq X$ is closed and $x \in X \backslash F$, then $x$ and $F$ have two disjoint zero-set neighborhoods in $Z_{c}(X)$. Consequently, there exist $g, h \in C_{c}(X)$ with $x \in X \backslash Z(h) \subseteq Z(g) \subseteq F^{c}$.

REMARK 4.2. $X$ is a $c$-completely regular space if and only if $F=\left\{Z(f): f \in C_{c}(X)\right\}$ is a base for the closed sets in $X$ or equivalently if and only if $B=\left\{\operatorname{int}(Z(f)): f \in C_{c}(X)\right\}$ is a base for the open sets in $X$.

If we apply the proof of $[9,3.11(\mathrm{a})]$ word-for-word, we obtain the following.

Proposition 4.3. Let $X$ be a c-completely regular space and $A, B$ be two disjoint closed sets in $X$ such that $A$ is compact, then there is $f \in C_{c}(X)$ with $f(A)=0, f(B)=1$.

The following fact which incidentally shows the previous result holds if we replace $C_{c}(X)$ by $C(X, K)$, where $K$ is a countable subfield of $\mathbb{R}$, is crucial in our study and although its proof is routine, we present some part of it for the sake of the reader.

Proposition 4.4. Let $X$ be a topological space. Then the following statements are equivalent.

1. $X$ is a zero-dimensional space (i.e., a $T_{1}$-space with a base consisting of clopen sets).

2. For each closed subset $F$ of $X$ and $x \in X \backslash F$, there exists $f \in C^{F}(X)$ with $f(F)=0$ and $f(x)=1$.

3. For each closed subset $F$ of $X$ and $x \in X \backslash F$, there is $f \in A$ such that $f(F)=0$ and $f(x)=1$, where $A$ is a subring of $C(X)$ whose elements take values in a countable subring of $\mathbb{R}$.

4. $X$ is c-completely regular space.

Proof. We only show that (4) $\Rightarrow(1)$. Let $x \in X$ and $U_{x}$ be a neighborhood of $x$. Then there exists $f \in C_{c}(X)$ with $f(x)=1, f\left(U_{x}^{c}\right)=0$ and $f(X) \subseteq[0,1]$. Since $f \in C_{c}(X)$, we infer that there exists $r \in[0,1]$ such that $r \notin f(X)$. Hence $x \in f^{-1}(r, \infty)=f^{-1}([r, \infty)) \subseteq U_{x}$. But clearly $A_{x}=f^{-1}(r, \infty)=f^{-1}([r, \infty))$ is a clopen set and we are done.

Corollary 4.5. Let $X$ be a Hausdorff space, then $X$ is a zerodimensional space if and only if its topology coincides with the weak 
topology induced by $C_{c}(X)\left(C^{F}(X)\right)$ or by a subring of $C_{c}(X)$ such as $A$ in part (3) of the above proposition.

It is well-known that as far as the algebraic properties of $C(X)$ are concerned we may always assume that $X$ is a completely regular space. In what follows we similarly observe that as far as algebraic (lattice) properties of $C_{c}(X)\left(C^{F}(X)\right)$ or some other natural subrings of $C_{c}(X)$ are to be investigated, we may impose the blanket assumption of the zero-dimensionality on $X$.

THEOREM 4.6. Let $X$ be any space (not necessarily completely regular). Then there is a zero-dimensional space $Y$ which is a continuous image of $X$ and $C_{c}(X) \cong C_{c}(Y)$.

Proof. We define $x \sim x^{\prime}$ on $X$ if $f(x)=f\left(x^{\prime}\right)$ for all $f \in C_{c}(X)$. Clearly, this is an equivalence relation on $X$. Put $Y=\{[x]: x \in X\}$, where $[x]$ is the equivalence class of $x \in X$. We define $\varphi: X \rightarrow Y$ by $\varphi(x)=[x]$. Now for each $f \in C_{c}(X)$, let us define $g_{f} \in \mathbb{R}^{Y}$ by $g_{f}(y)=f(x)$, where $y=[x]$, hence $f=g_{f} \circ \varphi$. Now put $C^{\prime}=\left\{g_{f}: f \in C_{c}(X)\right\} \subseteq \mathbb{R}^{Y}$ and we observe that $g \in C^{\prime}$ if and only if $g \circ \varphi=f$ for some $f \in C_{c}(X)$. Let us now consider the weak topology on $Y$ which is induced by $C^{\prime}$. Therefore $C^{\prime} \subseteq C(Y)$ and since for each open set $G$ in $\mathbb{R}$ we have $\varphi^{-1}\left(g^{-1}(G)\right)=f^{-1}(G)$, we infer that $\varphi$ is continuous. We also note that for $y, y^{\prime} \in Y$ with $y \neq y^{\prime}$, there exists $g \in C^{\prime}$ with $g(y) \neq g\left(y^{\prime}\right)$, hence $Y$ is Hausdorff. It is also manifest, by definition, that for each $g_{f} \in C^{\prime}$ we have $\left|g_{f}(Y)\right|=|f(X)| \leq \aleph_{0}$. Finally, it is trivial to see that $\theta: C_{c}(Y) \rightarrow C_{c}(X)$, where $\theta(g)=g \circ \varphi$ is an isomorphism. Hence it remains to be shown that $Y$ is zero-dimensional. To this end, let $H \subseteq Y$ be an open set and $y \in H$. Then $y \in g_{1}^{-1}\left(a_{1}, b_{1}\right) \cap g_{2}^{-1}\left(a_{2}, b_{2}\right) \cap \ldots \cap g_{n}^{-1}\left(a_{n}, b_{n}\right) \subseteq H$ for some $g_{1}, g_{2}, \ldots, g_{n} \in C^{\prime}, a_{i}, b_{i} \in \mathbb{R}, i=1,2, \ldots, n$. Since for each $g_{i}$ we have $\left|g_{i}(Y)\right| \leq \aleph_{0}$, we infer that without loss of generality we may assume that $a_{i}, b_{i} \notin g_{i}(Y), \quad i=1,2, \ldots, n$. Consequently, $y \in g_{1}^{-1}\left(a_{1}, b_{1}\right) \cap g_{2}^{-1}\left(a_{2}, b_{2}\right)$ $\cap \ldots \cap g_{n}^{-1}\left(a_{n}, b_{n}\right)=g_{1}^{-1}\left[a_{1}, b_{1}\right] \cap g_{2}^{-1}\left[a_{2}, b_{2}\right] \cap \ldots \cap g_{n}^{-1}\left[a_{n}, b_{n}\right] \subseteq H$, hence $y \in D_{y} \subseteq H$, where $D_{y}=g_{1}^{-1}\left(a_{1}, b_{1}\right) \cap g_{2}^{-1}\left(a_{2}, b_{2}\right) \cap \ldots \cap g_{n}^{-1}\left(a_{n}, b_{n}\right)$ is a clopen set and we are done.

REMARK 4.7. In the previous proof we note that if $C(X, K)$ is a subring of $C_{c}(X)$ whose elements take values in a countable subring $K$ of $\mathbb{R}$, then $\theta(C(X, K))=C(Y, K)$, i.e., $C(X, K) \cong C(Y, K)$. In particular, $C_{r}(X) \cong C_{r}(Y)$, $C_{i}(X) \cong C_{i}(Y)$ and one can also easily show that $C^{F}(X) \cong C^{F}(Y)$. 
The following interesting result shows that if $X$ is a space whose class of components is not large, then $C_{c}(X)$ is $C(Y)$ for some zero-dimensional space $Y$.

COROLLARY 4.8. Let $X$ be a topological space whose set of components is countable. Then $C_{c}(X) \cong C(Y)$ for some zero-dimensional space $Y$.

Proof. In view of the previous theorem there exists a zero-dimensional space $Y$ such that $Y$ is a continuous image of $X$ and $C_{c}(X) \cong C_{c}(Y)$. But it is clear that $Y$ is totally disconnected (i.e., the only nonempty connected subsets of $Y$ are the singletons). Consequently, $Y$ must be countable and therefore $C_{c}(X) \cong C_{c}(Y)=C(Y)$.

Corollary 4.9. Let $X$ be a topological space with only $n$ components. Then $C_{c}(X)=C^{F}(X) \cong \prod_{i=1}^{n} R_{i}$, where $R_{i}=\mathbb{R}$ for $i=1,2, \ldots, n$. Moreover $C(X, K) \cong \prod_{i=1}^{n} K_{i}$, where $K_{i}=K$ for $i=1,2, \ldots, n$ and $K$ is a countable subring of $\mathbb{R}$, hence $C_{r}(X) \cong \prod_{i=1}^{n} Q_{i}$, where $Q_{i}=\mathrm{Q}$ and $C_{i}(X) \cong \prod_{i=1}^{n} Z_{i}$,
where $Z_{i}=Z$.

\section{Countably $P$-space}

In this section we introduce countably $P$-spaces. We recall that $X$ is a $P$ space if and only if $C(X)$ is a regular ring, see [9, $4 \mathrm{~J}]$. We observe trivially if $C(X)$ is regular, then so too is $C_{c}(X)$, but the converse is not true in general. Motivated by this, we offer the following definition.

Definition 5.1. A space $X$ is called a countably $P$-space (briefly, $C P$ space) if $C_{c}(X)$ is regular.

Example 5.2. Let $X=[0,1] \cup \mathbb{N}$ be the free sum of $[0,1]$ and the natural numbers $\mathbb{N}$. Clearly, $X$ is a $C P$-space which is not a $P$-space.

Proposition 5.3. Every P-space is a CP-space.

Proof. Let $X$ be a $P$-space. We must show that for each $f \in C_{c}(X)$, there exists $g \in C_{c}(X)$ with $f=f^{2} g$. Since $C(X)$ is regular, there is $h \in C(X)$ with $f=f^{2} h$. Consequently, $f=f^{2} g$, where $g=h^{2} f$. It is also evident that 

$Z(f) \subseteq Z(g)$ and $g(x)=\frac{1}{f(x)}$, whenever $x \notin Z(f)$. Hence $g \in C_{c}(X)$ and we
are done.

REMARK 5.4. Lemma 2.4, gives another proof of the above result, for $Z(f)=Z\left(f^{2}\right)$ for all $f \in C_{c}(X)$, hence $f=f^{2} g$ for some $g \in C_{c}(X)$.

It is well-known that $X$ is a $P$-space if and only if every $G_{\delta}$-set is open, see $[9,4 \mathrm{~J}(3)]$. The following which plays a crucial role in our study is the counterpart of the previous fact in $C_{c}(X)$.

THEOREM 5.5. A space $X$ is a CP-space if and only if every zero-set in $Z_{c}(X)$ is open. Moreover, in this case whenever $\left\{f_{i}\right\}_{i=1}^{\infty}$ is a countable set in $C_{c}(X), \bigcap_{i=1}^{\infty} Z\left(f_{i}\right)$ is an open zero-set in $Z_{c}(X)$.

Proof. Let $X$ be a $C P$-space and $f \in C_{c}(X)$, then $f=f^{2} g$ for some $g \in C_{c}(X)$. Clearly, $e=f g$ is an idempotent in $C_{c}(X)$ and $Z(f)=Z(e)$ is open. Conversely, if $Z(f)$ is open for all $f \in C_{c}(X)$, we are through by Remark 5.4. Finally, let $I$ be the ideal in $C_{c}(X)$ generated by $\left\{f_{i}\right\}_{i=1}^{\infty}$, i.e., $I=\sum_{i=1}^{\infty} f_{i} C_{c}(X)$. Since $C_{c}(X)$ is regular, one can write $I=\sum_{i=1}^{\infty} \oplus e_{i} C_{c}(X)$, where each $e_{i}$ is an idempotent in $C_{c}(X)$ and $e_{i} e_{j}=0$ for all $i \neq j$, see [13, Lemma 2] or [7, the proof of Proposition 1.4]. We note that whenever $e_{j}(x) \neq 0$ for some $x \in X$, then $e_{i}(x)=0$ for all $i \neq j$. Clearly, $\bigcap_{i=1}^{\infty} Z\left(f_{i}\right)=\bigcap_{g \in I} Z(g)=\bigcap_{i=1}^{\infty} Z\left(e_{i}\right)$. Now we define $g=\sum_{i=1}^{\infty} \frac{e_{i}}{2^{i}\left(1+e_{i}\right)}$ and it is evident $g \in C(X), Z(g)=\bigcap_{i=1}^{\infty} Z\left(e_{i}\right)$. Hence if we show that $g \in C_{c}(X)$, then we are done. But for each $x \in X$ there exists at most a unique $i \geq 1$ such that $e_{i}(x) \neq 0$, therefore $g(x)=\frac{e_{i}(x)}{2^{i}\left(1+e_{i}(x)\right)}=\frac{1}{2^{i+1}}$ and consequently $g(X) \subseteq\left\{0, \frac{1}{2^{2}}, \frac{1}{2^{3}}, \frac{1}{2^{4}}, \ldots\right\}$, i.e., $g \in C_{c}(X)$.

REMARK 5.6. Let $X$ be a $C P$-space. Then $C^{F}(X)(C(X, K)$, where $K$ is a countable subfield of $\mathbb{R}$ and in particular $\left.C_{r}(X)\right)$ is a regular ring too. To see this, one can just apply the proof of either Proposition 5.3 or Remark 5.4, verbatim. We should remind the reader that the previous theorem which is basic in our study (see for example, Remark 6.10) may not be true for $C^{F}(X)$, but our proof shows that it is in fact true for the subring $C(X, K)$ and in particular for $C_{r}(X)$. Finally we must also emphasize that it is trivial 
to see that each regular subring of $C(X)$ should contain an isomorphic copy of $\mathrm{Q}$, hence $C_{i}(X)$ can never be regular.

Corollary 5.7. Let $X$ be a zero-dimensional CP-space. Then every $G_{\delta}$-set $A$ containing a compact set $S$ contains a zero-set in $Z_{c}(X)$ containing S. In particular, every zero-dimensional CP-space is a P-space.

Proof. Let $A=\bigcap_{n=1}^{\infty} U_{n}$, where each $U_{n}$ is an open set. Since $A \supseteq S$, we infer that $S$ and $X \backslash U_{n}$ are disjoint for all $n \geq 1$. Thus by Proposition 4.3, there exists a zero-set $F_{n} \in Z_{c}(X)$ with $S \subseteq F_{n} \subseteq U_{n}$ for all $n \geq 1$, $S \subseteq \bigcap_{n=1}^{\infty} F_{n}$. But by the previous theorem $\bigcap_{n=1}^{\infty} F_{n}=Z(f)$ for some $f \in C_{c}(X)$ and we are through, for $S \subseteq Z(f) \subseteq A$. The final part is now immediate.

Next, let us put $O_{p}^{c}=O_{p} \cap C_{c}(X)$ and $M_{p}^{c}=M_{p} \cap C_{c}(X)$, where $p \in X$ and $O_{p}$ is the ideal of $C(X)$ consisting of all $f$ in $C(X)$ for which $Z(f)$ is a neighborhood of $p$. It goes without saying that $M_{p}^{c}$ is a maximal ideal in $C_{c}(X)$ and $O_{p}^{c}$ is a $z_{c}$-ideal in $C_{c}(X)$. The following is now the counterpart of $[9,4 \mathrm{~J}]$ for $C_{c}(X)$ and for its proof it suffices to apply the corresponding proof for $C(X)$ word-for word and also invoke the previous theorem.

THEOREM 5.8. Let $X$ be a topological space. Then the following statements are equivalent.

1. $X$ is a CP-space.

2. $C_{c}(X)$ is a regular ring.

3. Each ideal in $C_{c}(X)$ is a $z_{c}$-ideal.

4. Each prime ideal in $C_{c}(X)$ is a maximal ideal.

5. For each $p \in X, M_{p}^{c}=O_{p}^{c}$.

6. Every zero-set in $Z_{c}(X)$ is open.

7. Each ideal in $C_{c}(X)$ is an intersection of maximal ideals.

8. For all $f, g \in C_{c}(X),(f, g)=\left(f^{2}+g^{2}\right)$.

9. For every $f \in C_{c}(X), Z(f)(X \backslash Z(f))$ is $C$-embedded.

10. If $\left\{f_{i}: i \in \mathbb{N}\right\} \subseteq C_{c}(X)$, then $\bigcap_{i=1}^{\infty} Z\left(f_{i}\right)$ is an open zero-set in $Z_{c}(X)$.

Finally, we conclude this section with the following remarks.

REMARK 5.9. Let $X$ be any $C P$-space. Then by Theorem 4.6 and Corollary 5.7, there is a $P$-space $Y$ which is a continuous image of $X$. 
REMARK 5.10. In Theorem 5.8, if we replace $z_{c}$-ideal by $z_{F}$-ideal and put $O_{p}^{F}=O_{p} \cap C^{F}(X)$ and $M_{p}^{F}=M_{p} \cap C^{F}(X)$, then all the equivalences (2)(9) in this theorem are valid for $C^{F}(X)$. But we should also remind the reader that all the previous equivalences (2)-(10) are true with the same proofs for $C(X, K)$, where $K$ is a countable subfield of $\mathbb{R}$ and in particular for $C_{r}(X)$. It is also emphasized that whenever $X$ is a zero-dimensional space the proof of Corollary 5.7, shows that for a countable subfield $K$ of $\mathbb{R}$, $C_{c}(X)(C(X))$ is regular if and only if $C(X, K)$ is regular. As for $C_{i}(X)$, we recall that $C_{i}(X)$ can never be a regular ring.

\section{6. $C_{c}(X)$ vs. $C(X)$}

A commutative ring $R$ is called self-injective ( $\aleph_{0}$-selfinjective) if every homomorphism from an ideal (countably generated ideal) of $R$ into $R$ can be extended from $R$ into $R$. Commutative regular rings, in general, are rarely $\aleph_{0}$-selfinjective, see [10, Example 14.7]. But surprisingly in [7, Theorem 1], it is shown that $C(X)$ is regular if and only if $C(X)$ is $\aleph_{0}$-selfinjective. Another interesting property of $C(X)$ is the fact that whenever the set of isolated points is dense in $X$, then $C(X)$ is a self-injective ring if and only if $C(X)$ is isomorphic to a direct product of fields, see [3, Theorem 4.2, Proposition 4.4]. We also recall that if $C(X)$ satisfies the latter condition, then it is in fact isomorphic to a direct product of the field of the real numbers, that is to say, $C(X)$ keeps its faithfulness to the real numbers. In this section, we show that $C_{c}(X)$ enjoys all these interesting properties too. We should also remind the reader that $C^{*}(X)$ can never have any of these properties, unless $X$ is a finite space, in which case, $C(X)=C^{*}(X)=C_{c}(X)=C^{F}(X)=C_{F}(X) \cong \prod_{i \in I} R_{i}$, where $R_{i}=\mathbb{R}$ and $|I|=|X|$ and clearly $C_{r}(X) \cong \prod_{i \in I} Q_{i}$, where $Q_{i} \stackrel{i \in I}{=} \mathrm{Q}$, $C_{i}(X) \cong \prod_{i \in I} Z_{i}$, where $Z_{i}=Z_{\text {. }}$

Before observing the validity of the last property mentioned above for $C_{c}(X)$, we need the next lemma.

LEMMa 6.1. Let $0 \neq I$ be an ideal in $C_{c}(X)(C(X))$ which is a field as a ring. Then $I \cong \mathbb{R}$. Similarly, if $0 \neq I$ is an ideal in $C(X, K)$ which is a field, where $K$ is a subfield of $\mathrm{R}$, then $I \cong K$.

Proof. Since $I$ is a field, it has an identity, $e \in C_{c}(X)$ say. Hence $0 \neq e=e^{2}$ is an idempotent and $e C_{c}(X) \subseteq I$, for $I$ is an ideal in $C_{c}(X)$. Clearly $e C_{c}(X)$ is an ideal in $I$ too, hence $e C_{c}(X)=I$ (note, $I$ is a field). It is also 
evident that every ideal of $C_{c}(X)$ which is inside $I$, is an ideal of $I$ too. Hence $I$ is the only nonzero ideal of $C_{c}(X)$ which is in $I$. Consequently, $I$ is a minimal ideal in $C_{c}(X)$. Now $e C_{c}(X) \cong C_{c}(X) /(1-e) C_{c}(X)$ implies that $(1-e) C_{c}(X)$ must be a maximal ideal in $C_{c}(X)$. Let $x \in Z(1-e)$ and define $\varphi: C_{c}(X) \rightarrow \mathbb{R}$ by $\varphi(f)=f(x)$, hence $C_{c}(X) / \operatorname{ker} \varphi \cong \mathbb{R}$. But clearly $(1-e) C_{c}(X) \subseteq \operatorname{ker} \varphi$ and therefore $(1-e) C_{c}(X)=\operatorname{ker} \varphi$, by the maximality of $(1-e) C_{c}(X)$. Thus $I=e C_{c}(X) \cong C_{c}(X) / \operatorname{ker} \varphi \cong \mathbb{R}$, and we are done. Finally, this proof can be carried over verbatim for $C(X)$ and $C(X, K)$.

REMARK 6.2. The above proof shows that if $I$ is a nonzero minimal ideal in $C(X)$ or in either $C_{c}(X)$ or $C^{F}(X)$ (resp. $C(X, K)$, where $K$ is a subfield of $\mathbb{R}$ ), then we must have $I \cong \mathbb{R}$ (resp. $I \cong K$ ). It is interesting to note that if in the ring $C(X, K)$ we assume that $K$ is just a subring of $\mathbb{R}$ which is not a field, then $C(X, K)$ cannot contain a nonzero minimal ideal. In particular, the socle of $C_{i}(X)$ is zero.

Corollary 6.3. Let $\prod_{i \in I} F_{i} \stackrel{\varphi}{\cong} C_{c}(X)$, where every $F_{i}$ is a field. Then $F_{i} \cong \mathbb{R}$ for all $i \in I$. Similarly, if $\prod_{i \in I} F_{i} \stackrel{\varphi}{\cong} C(X, K)$, where every $F_{i}$ is a field and $K$ is a subfield of $\mathbb{R}$, then $F_{i} \cong K$ for all $i \in I$.

Proof. We may consider $F_{i}$ as $F_{i}=\left\{\left\langle a_{j}\right\rangle: a_{i} \in F_{i}, a_{j}=0\right.$, for all $j \neq i\}$. Hence each $F_{i}$ is an ideal in $\prod_{i \in I} F_{i}$ which is also a field. Consequently, $\varphi\left(F_{i}\right)=A_{i} \subseteq C_{c}(X)$ is an ideal which is also a field in $C_{c}(X)$ for all $i \in I$. Hence, by the previous lemma we have $A_{i} \cong \mathbb{R}$, i.e., $F_{i} \cong A_{i} \cong \mathbb{R}$ and we are done. The proof for $C(X, K)$ is similar.

REMARK 6.4. One can clearly observe that $C_{i}(X)$ can never be isomorphic to any direct product of fields, for $C_{i}(X)$ cannot be a regular ring . More generally, in view of the previous remark, no direct product of fields can be even a direct summand of $C_{i}(X)$.

It is well-known and easy to see that if $I$ is an ideal in a commutative reduced ring $R$, then $I$ is essential in $R$ (i.e., $I$ intersects every nonzero ideal nontrivially) if and only if $\operatorname{Ann}(I)=\{r \in R: r I=0\}=0$, see also [16]. Using this trivial fact we prove the following interesting result.

\section{THEOREM 6.5. The following statements are equivalent.}

1. $C_{c}(X)$ is a selfinjective ring and the set of isolated points of $X$ is dense in $X$. 
2. $C_{c}(X) \cong \prod_{i \in I} R_{i}$, where $R_{i}=\mathbb{R}$ for all $i \in I$ and every nonzero ideal of $C(X)$ intersects $C_{c}(X)$ nontrivially and $C(X), C_{c}(X)$ have the same socle.

Proof. $\quad(1) \Rightarrow(2)$. Let $C_{F}(X)=\sum_{k \in K} \oplus e_{k} C(X)$, where $e_{k}=e_{k}^{2}$ and each $e_{k} C(X)$ is a minimal ideal in $C(X)$, be the socle of $C(X)$, see [16]. By the comment preceding Remark 2.2, $C_{F}(X) \subseteq C_{c}(X)$. Clearly $e_{k} C(X)=e_{k} C_{c}(X)$ for each $k \in K$. Since $C(X)=e_{k} C(X) \oplus\left(1-e_{k}\right) C(X)$, we infer that each ideal of $C_{c}(X)$ inside $e_{k} C(X)$ is also an ideal of $C(X)$. Hence, each $e_{k} C(X)$ is a minimal ideal of $C_{c}(X)$ and therefore $C_{F}(X) \subseteq C_{c F}(X)$, where $C_{c F}(X)$ is the socle of $C_{c}(X)$. Inasmuch as the set of isolated points of $X$ is dense in $X$ we have $\operatorname{Ann}\left(C_{F}(X)\right)=0$ in $C(X)$, by [16, Proposition 2.1], a fortiori, $\operatorname{Ann}\left(C_{F}(X)\right)=0$ in $C_{c}(X)$. By the above comment, we note that $C_{F}(X)$ is essential in both $C(X)$ and $C_{c}(X)$. This incidentally also shows that every nonzero ideal of $C(X)$ intersects $C_{c}(X)$ nontrivially. We now claim that $C_{F}(X)=C_{c F}(X)$. To see this, we recall that in any commutative ring $R$ every ideal inside the socle of $R$ is a direct summand of the socle, see [16], hence we must have $C_{c F}(X)=C_{F}(X) \oplus A$, where $A$ is an ideal in $C_{c}(X)$. Now by the essentiality of $C_{F}(X)$ we infer that $A=0$ and we are done. Finally, let us define $\varphi: C_{c}(X) \rightarrow \prod_{k \in K} e_{k} C_{c}(X)=R$ by $\varphi(f)=\left\langle e_{k} f\right\rangle$. Since $\operatorname{Ann}\left(C_{c F}(X)\right)=0$, we infer that $\varphi$ is one-one. Next, we claim that $\varphi$ is also onto. To see this, let $\left\langle e_{k} f_{k}\right\rangle \in R$, where $f_{k} \in C_{c}(X)$ for all $k \in K$ and we are to find $f \in C_{c}(X)$ with $\varphi(f)=\left\langle e_{k} f\right\rangle=\left\langle e_{k} f_{k}\right\rangle$. Put $\lambda=\left\langle f_{k}\right\rangle \in \prod_{k \in K} T_{k}$, where $T_{k}=C_{c}(X)$ for all $k \in K$. Now by considering $C_{F}(X)$ as an ideal in $\prod_{k \in K} T_{k}$, we define $\theta: C_{F}(X) \rightarrow C_{c}(X)$ by $\theta(x)=\lambda x$ for all $x \in C_{F}(X)$ (note, in fact $\theta$ is defined by $\theta\left(e_{k}\right)=e_{k} f_{k}$ for all $\left.k \in K\right)$. Now by the selfinjectivity of $C_{c}(X)$, $\theta$ can be extended to $\theta^{*}: C_{c}(X) \rightarrow C_{c}(X)$. Hence $\theta(g)=\theta^{*}(g)=g f$ for all $g \in C_{F}(X)$, where $f=\theta^{*}(1) \in C_{c}(X)$. Thus $\theta\left(e_{k}\right)=e_{k} f$, hence $\theta\left(e_{k}\right)=e_{k} f=$ $e_{k} f_{k}$ for all $k \in K$ and consequently $\varphi(f)=\left\langle e_{k} f\right\rangle=\left\langle e_{k} f_{k}\right\rangle$ and we are done. Thus we have already shown that $\varphi: C_{c}(X) \rightarrow \prod_{k \in K} e_{k} C_{c}(X)$ is an isomorphism. But each $R_{k}=e_{k} C_{c}(X)$ is a minimal ideal in $C_{c}(X)$ which is also a ring with $e_{k}$ as its identity. Since each ideal of $R_{k}$ is also an ideal of $C_{c}(X)$ (note, $\left.C_{c}(X)=e_{k} C_{c}(X) \oplus\left(1-e_{k}\right) C_{c}(X)\right)$, we infer that $R_{k}$ cannot have a nonzero ideal (note, $R_{k}$ is a minimal ideal in $C_{c}(X)$ ). Consequently, $R_{k}$ is a field and in view of Lemma 6.1, we must have $R_{k} \cong \mathbb{R}$ for all $k \in K$ and this completes the proof.

$(2) \Rightarrow(1)$. It is well-known that the direct product of any collection of fields is a selfinjective ring, see also [12]. It remains to be shown that the set of isolated points of $X$ is dense in $X$. But by [16, Proposition 2.1], the density 
of these points in $X$ is equivalent to $\operatorname{Ann}\left(C_{F}(X)\right)=0$ in $C(X)$. Clearly, $\sum_{i \in I} \oplus R_{i}$ is the socle of $\prod_{i \in I} R_{i}$ and it is essential in $\prod_{i \in I} R_{i}$. This immediately implies that $C_{F}(X)$ is essential in $C_{c}(X)$ and therefore $\operatorname{Ann}\left(C_{F}(X)\right)=0$ in $C_{c}(X)$, for $C_{c}(X)$ is reduced. We now claim that $\operatorname{Ann}\left(C_{F}(X)\right)=0$ in $C(X)$ too. If not, then $C_{c}(X) \cap \operatorname{Ann}_{C(X)}\left(C_{F}(X)\right)=\operatorname{Ann}_{C_{c}(X)}\left(C_{F}(X)\right) \neq 0$, which is absurd. Hence as we observed earlier $\operatorname{Ann}\left(C_{F}(X)\right)=0$ in $C(X)$ implies that the set of isolated points of $X$ is dense in $X$.

REMARK 6.6. If we replace $C_{c}(X)$ by $C^{F}(X)$ in the previous theorem, then using the same proof without the slightest change, the result remains valid for $C^{F}(X)$ too. But the result fails for $C(X, K)$, where $K$ is any countable subring of $\mathbb{R}$, for $C(X, K)$ can never contain the nonzero socle of $C(X)$. In particular, it also fails for $C_{r}(X), C_{i}(X)$.

Before proving the remaining property for $C_{c}(X)$, let us recall that a subset $S$ of a commutative ring $R$ is said to be orthogonal, provided $x y=0$ for all $x, y \in S$ with $x \neq y$. If $S \cap T=\varnothing$ and $S \cup T$ is an orthogonal set in $R$, then $a \in R$ is said to separate $S$ from $T$ if $s^{2} a=s$ for all $s \in S$ and $a \in \operatorname{Ann}(T)$, see [12], [14] for more details. We recall the following fact which is in [12, Theorem 2.2] and [14, Proposition 1.2].

LEMma 6.7. Let $R$ be a reduced ring (not necessarily commutative). Then the following statements are equivalent.

1. $R$ is selfinjective ( $\aleph_{0}$-selfinjective).

2. $R$ is regular and whenever $S \cup T$ is an orthogonal (countable orthogonal) set with $S \cap T=\varnothing$, then there is an element in $R$ which separates $S$ from $T$.

We also need the following fact.

LEMma 6.8. The following statements are equivalent in $C_{c}(X)(C(X))$.

1. If $S \cup T$ is an orthogonal set in $C_{c}(X)(C(X))$ with $S \cap T=\varnothing$, then there exists $f \in C_{c}(X)(f \in C(X))$ which separates $S$ from $T$.

2. For any orthogonal set $L$ in $C_{c}(X)(C(X))$ there exists $f \in C_{c}(X)$ $(f \in C(X))$ with $g^{2} f=g$ for all $g \in L$.

Proof. We only give a proof for $C_{c}(X)$ (note, the same proof works for $C(X)$ ). 
$(1) \Rightarrow(2)$. It is evident.

$(2) \Rightarrow(1)$. We are to show that there exists $h \in C_{c}(X)$ such that $h T=0$ and $g^{2} h=g$ for all $g \in S$. By (2), there exists $f_{1} \in C_{c}(X)$ with $g^{2} f_{1}=g$ for all $g \in S \cup T$. We also note that $S \cup(2 T)$ is an orthogonal set, where $2 T=\{2 t: t \in T\}$. Hence there exists $f_{2} \in C_{c}(X)$ with $g^{2} f_{2}=g$ for all $g \in S \cup(2 T)$. For each $g \in T$ we have $4 g^{2} f_{1}=4 g$ and $4 g^{2} f_{2}=(2 g)^{2} f_{2}=2 g$. Now put $h=2 f_{2}-f_{1} \in C_{c}(X)$, hence $4 g^{2} h=0$ and therefore $g^{2} h=0=(g h)^{2}$, i.e., $g h=0$ for all $g \in T$. Finally, if $g \in S$, then $g^{2} h=2 g^{2} f_{2}-g^{2} f_{1}=2 g-g=g$ and this completes the proof.

REMARK 6.9. By applying the proof of the previous result one can easily show that this lemma is also true in any reduced ring $R$ (not necessarily commutative) with $2 x \neq 0$ for all $0 \neq x \in R$, see also [25]. We should also remind the reader that in the previous lemma, we may replace $S, T$ and $L$ by countable orthogonal sets.

Theorem 6.10. Let $X$ be a topological space. Then $C_{c}(X)$ is a regular ring if and only if $C_{c}(X)$ is an $\aleph_{0}$-selfinjective ring.

Proof. If $C_{c}(X)$ is $\aleph_{0}$-selfinjective, then $C_{c}(X)$ is regular, by Lemma 6.7. Conversely, by Lemma 6.7, and Remark 6.9, it suffices to show that whenever $S$ is a countable orthogonal set in $C_{c}(X)$, then there exists $f \in C_{c}(X)$ with $g^{2} f=g$ for all $g \in S$. To see this, let $S=\left\{f_{i}\right\}_{i=1}^{\infty}$, where $f_{i} \neq 0$ for all $i \in I$. Since $C_{c}(X)$ is regular and in view of Theorem 5.5, we infer that $\bigcap_{i=1}^{\infty} Z\left(f_{i}\right)=Z(h)$ is an open set, where $h \in C_{c}(X)$. Now we define $G_{i}=X \backslash Z\left(f_{i}\right)$ for all $i \geq 1$. Since $f_{i} f_{j}=0$, we have $G_{i} \cap G_{j}=\varnothing$ for all $i \neq j$, and we also note that each $G_{i}$ is a clopen set. Finally, we define $G=\bigcup_{i=1}^{\infty} G_{i}$ and hence $X=\left(\bigcup_{i=1}^{\infty} G_{i}\right) \bigcup(X \backslash G)$. Therefore we may define $f: X \rightarrow \stackrel{i=1}{R}$ by $f(x)=\left\{\begin{array}{cl}f_{i}(x) & , x \in G_{i} \\ 0 & , x \notin G\end{array}\right.$, i.e., $\left.f\right|_{G_{i}}=f_{i}$ for all $i \geq 1$ and $f(x)=0$ for all $x \notin G$, hence $f$ is continuous by $[9,1 \mathrm{~A}(2)]$. We also note that $|f(X)| \leq$ $\left|\bigcup_{i=1}^{\infty} f_{i}(X)\right| \leq \aleph_{0}$, hence $f \in C_{c}(X)$. Clearly, $f(x)=f_{i}(x)$ for all $x \in G_{i}=$ $X \backslash Z\left(f_{i}\right)$ and $Z\left(f_{i}\right) \subseteq Z(f)$ for each $i \geq 1$, hence $f f_{i}=f_{i}^{2}$ for all $f_{i} \in S$ and we are done.

REMARK 6.11. We should emphasize that Theorem 6.10, is not true for $C^{F}(X)$ in general. The reason that the above proof does not work for $C^{F}(X)$ 
lies in the fact that the second part of Theorem 5.5, may not be true for $C^{F}(X)$, see Example 7.1, Remark 7.4. But we should remind the reader that the proof of Theorem 6.10 , can be applied word-for-word to show that the theorem is also true for $C(X, K)$, where $K$ is a subfield of $\mathbb{R}$, and in particular for $C_{r}(X)$. But as we observed earlier $C_{i}(X)$ is never a regular ring, a fortiori is never $\aleph_{0}$-selfinjective.

\section{7. $C_{c}(X)$ may not be a $C(Y)$}

We have already shown that whenever $C(X)$ is $\aleph_{0}$-selfinjective (note, $C(X)$ is regular too), then so too is $C_{c}(X)$. In this section we show that $C(X)$ may be selfinjective, but $C_{c}(X)$ may not be so. We also observe that whenever $C(X)$ is $\aleph_{0}$-selfinjective, then not every regular subring of $C(X)$ is $\aleph_{0}$-selfinjective (we should emphasize that in this case $C_{c}(X)$ is both regular and $\aleph_{0}$-selfinjective). Finally we present an example of a space $X$ for which $C_{c}(X)$ is not isomorphic to any $C(Y)$. In the following example, we see that $C(X)=C_{c}(X)$ is selfinjective, but $C^{F}(X)$ is not even $\aleph_{0}$-selfinjective. We should remind the reader that all three rings are regular.

EXAmple 7.1. Let $X$ be an infinite countable discrete space. We may put $C(X)=\prod_{i=1}^{\infty} R_{i}$, where $R_{i}=\mathbb{R}$ for all $i \geq 1$. Hence $C(X)=C_{c}(X)$ is a selfinjective regular ring. Finally, we claim that $C^{F}(X)$, although a regular subring of $C(X)$, is not $\aleph_{0}$-selfinjective. To see this, put

$$
R=\left\{\left\langle a_{i}\right\rangle \in \prod_{i=1}^{\infty} R_{i}: \text { there are only finitely many distinct } a_{i}\right\}
$$

Clearly, $R=C^{F}(X)$. Now put $X=A \cup B, A \cap B=\varnothing$, where $|A|=|B|=\aleph_{0}$. For each $i \in A$, let us define $f_{i}=\left\langle a_{i}\right\rangle \in C(X)$, where $a_{i}=r_{i} \neq 0, a_{j}=0$ for all $j \neq i$, moreover we assume that all $r_{i}$ are distinct and for all $i \in B$, we define $g_{i}=\left\langle b_{i}\right\rangle \in C(X)$, where $b_{i}=1, b_{j}=0$ for all $j \neq i$. Clearly, if $S=\left\{f_{i}: i \in A\right\}, T=\left\{g_{j}: j \in B\right\}$, then $S \cap T=\varnothing$ and $S \cup T$ is an orthogonal set in $C^{F}(X)$. We claim that there is no element in $C^{F}(X)$ which separates $S$ from $T$. Let $h=\left\langle c_{i}\right\rangle \in C^{F}(X)$ separate $S$ from $T$ and obtain a contradiction. We must have $f_{i}^{2} h=f_{i}$ for all $i \in A$ and $g_{i} h=0$ for all $i \in B$. Consequently, we must have $c_{i}=\frac{1}{r_{i}}$ for all $i \in A$ and $c_{j}=0$ for all $j \in B$. Therefore $h \in C_{c}(X) \backslash C^{F}(X)$, which is the desired contradiction. 
Let us recall that a commutative ring $R$ is called a Baer ring if for any subset $S$ of $R$ we have $\operatorname{Ann}(S)=e R$, where $e=e^{2}$. A space $X$ is said to be extremally disconnected if every open set in $X$ has an open closure, see $[9,1 \mathrm{H}]$. In [3, Theorem 3.5] it is shown that $C(X)$ is a Baer ring if and only if $X$ is an extremally disconnected space.

For the proof of the following result, see [3, Theorem 4.2] and Lemma 6.7.

Theorem 7.2. Let $X$ be a topological space. Then the following statements are equivalent.

1. $C(X)$ is a Baer regular ring.

2. $X$ is an extremally disconnected $P$-space.

3. $C(X)$ is a selfinjective ring.

4. If $S \cup T$ is an orthogonal set in $X$ with $S \cap T=\varnothing$, then there exists an element in $C(X)$ which separates $S$ from $T$.

The next trivial result is needed.

LEMma 7.3. Let $T$ and $R$ be two commutative rings with $R \subseteq T$ and $R$ contains all idempotent elements in $T$. Then whenever $T$ is a Baer ring, so too is $R$.

Proof. Let $S \subseteq R$ be a subset, then $\operatorname{Ann}_{R}(S)=\operatorname{Ann}_{T}(S) \cap R$. But $\operatorname{Ann}_{T}(S)=e T, e^{2}=e \in T$, since $T$ is a Baer ring. Hence, by our assumption $e \in R$. Now it is clear that $e T \cap R=e R$ and we are through.

Finally we conclude this article by presenting the next two remarks, of which, the first one shows the validity of the title of this section and the second one gives some reasons for our interest in $C_{c}(X)$ and $C^{F}(X)$ and it also includes an open question. Incidentally, in the remark which follows, we observe that $C(X)$ and $C_{r}(X)$ (more generally $C(X, K)$, where $K$ is any field) are selfinjective, but neither $C_{c}(X)$ nor $C^{F}(X)$ are so. It is left to the reader to show that $C_{c}(X)$ is $\aleph_{0}$-selfinjective, but $C^{F}(X)$ is not, although it is a regular ring.

REMARK 7.4. There is a topological space $X$ such that there is no space $Y$ with $C_{c}(X) \cong C(Y)$. To see this, let $X$ be a discrete space which is uncountable (i.e., $|X|=|\mathbb{R}|$ ). Then $C(X) \cong \prod_{i \in X} R_{i}$, and $C_{r}(X) \cong \prod_{i \in X} Q_{i}$, where $R_{i}=\mathbb{R}, Q_{i}=\mathrm{Q}$ for all $i \in X$, are clearly selfinjective. Now let $X=A \cup B$, where $A, B$ are uncountable with $A \cap B=\varnothing$. There exists a one-one mapping $f$ of $X$ onto $\mathbb{R} \backslash\{0\}$. Now for each $i \in A$, let us put 
$f_{i}=\left\langle a_{i}\right\rangle \in C_{c}(X)$, where $a_{i}=f(i)$ and $a_{j}=0$ for all $j \neq i$, hence all components of $f_{i}$ are zero except one. For each $i \in B$, we also put $g_{i}=\left\langle b_{i}\right\rangle \in C_{c}(X)$ with $b_{i}=f(i)$ and $b_{j}=0$ for all $j \neq i$. Finally, we put $S=\left\{f_{i}: i \in A\right\}, T=\left\{g_{i}: i \in B\right\}$. It is evident that $S \cap T=\varnothing$ and $S \cup T$ is an orthogonal set in both $C_{c}(X)$ and $C^{F}(X)$. We now claim that there is no element in $C_{c}(X)$, a fortiori, in $C^{F}(X)$ which separate $S$ from $T$ (note, this shows that the latter two rings are not selfinjective, by Lemma 6.7). To see this, let $h \in C_{c}(X)$ be such an element and obtain a contradiction. We must have $g^{2} h=g$ for all $g \in S$, hence $h(i)=\frac{1}{f(i)}$ for all $i \in A$. But $f$ is one-one and $A$ is uncountable, hence $|h(X)|$ is uncountable which is a contradiction. Finally, we claim that the space $X$ is the one we promised at the outset of the remark. Since $X$ is an extremely disconnected $P$-space, we infer that $C(X)$ is a Baer regular ring, by Theorem 7.2. Hence, by Proposition 5.3, $C_{c}(X)$ is a regular ring and it is also a Baer ring, by Lemma 7.3. Finally we show that $C_{c}(X) \cong C(Y)$, where $Y$ is any space, leads us to a contradiction. To see this, we note that $C(Y)$ is a Baer regular ring too. Hence by Theorem 7.2, $Y$ is an extremally disconnected $P$-space and $C(Y)$ is a selfinjective ring. But we have already shown that $C_{c}(X)$ is not selfinjective and this is the desired contradiction.

REMARK 7.5. Let us put $A=C(X, K)$, where $K$ is a countable subring of $\mathbb{R}$. It is interesting to recall that when $K$ is a subfield of $\mathbb{R}$ and $X$ is a zero-dimensional space, then $X$ is a $P$-space if and only if $A$ is a regular ring, see Corollary 5.10 (note, in this case the subfield $K$ need not even be countable, see the proof of Proposition 5.3). Clearly there are infinitely many subrings such as $A$ in $C(X)$, but unfortunately none of them can be an $\mathbb{R}$-subalgebra of $C(X)$. But it seems when it comes to the study of $C(X)$ in general, then those subrings of $C(X)$ which are $\mathbb{R}$-subalgebras are more appropriate to be considered. Although for the above subring $A$ we may consider the $\mathbb{R}$-subalgebra $\mathbb{R}[A]$ (i.e., the $\mathbb{R}$ - algebra generated by $A$ over $\mathbb{R}$ ) of $C_{c}(X)$, but none of the nontrivial results (for example, Theorems 5.5, $5.8,6.5,6.10)$ in this article which are true for $C_{c}(X)$ may remain valid for $\mathbb{R}[A]$. In particular, when $X$ contains isolated points then the socle of $C(X)$ (i.e., $C_{F}(X)$ ) which is nonzero, see [2] and [16], appears naturally in both $C_{c}(X)$ and $C^{F}(X)$ too, but we must remind the reader that none of the previous subrings of $C(X)$ such as $A$ can contain $C_{F}(X)$ (note, these subrings cannot even contain any ideal of $C(X)$ containing a nonzero idempotent). Again for each subring such as $A$ one might put $A_{F}=A+C_{F}(X)$ to get the subring $A_{F}$ of $C_{c}(X)$ containing $C_{F}(X)$, but clearly $A_{F}$ and $C_{c}(X)$ 
cannot share the nontrivial results in this article (for example, even an easy and natural one such as Proposition 5.3, may not hold for $A_{F}$ ). Finally in contrast to $C_{c}(X)$ (for example, we may recall the interesting result of Rudin, Pelczynski and Semadeni in the introduction) some of its natural subrings such as $A$ and in particular $C_{r}(X)$ and $C_{i}(X)$ can never be isomorphic to any $C(Y)$, for otherwise they must contain an isomorphic copy of $\mathbb{R}$ which is impossible. With regard to the latter remark, the interested reader is encouraged to characterize all topological spaces $X$ (not necessarily compact) for which $C(X)=C_{c}(X)$ (resp. $C_{c}(X) \cong C(Y)$ for some space $Y$ ), an interesting question which is unsettled yet. But unfortunately it seems (at least to us) that these topological spaces cannot be easily described (note, compact scattered spaces have this property and there are uncountable non-compact spaces enjoying this property too, see also Corollary 4.8). All these facts and perhaps some others make us believe that among the subrings of $C(X)$ whose elements have countable image, $C_{c}(X)$ and $C_{F}(X)$ seem to be the natural companions for $C(X)$ which along with $C^{*}(X)$ can be considered in investigating the connections between the algebraic properties of $C(X)$ and the topological properties of $X$.

Acknowledgment. The authors would like to thank the well-informed referee of this article for the detailed report, corrections, and several constructive suggestions for improvement.

\section{REFERENCES}

[1] F. Azarpanah - O. A. S. Karamzadeh - A. Rezaei Aliabad, On $z^{\circ}$-ideals in $C(X)$, Fund. Math. 160 (1999), pp. 15-25.

[2] F. Azarpanah - O. A. S. Karamzadeh - S. Rahmati, $C(X)$ VS. $C(X)$ Modulo its socle, Colloq. Math. 3 (2008), pp. 315-336.

[3] F. Azarpanah - O. A. S. Karamzadeh, Algebraic characterization of some disconnected spaces, Italian. J. Pure Appl. Math. 12 (2002), pp. 155-168.

[4] F. Azarpanah - R. Mohamadian, $\sqrt{z}$-ideals and $\sqrt{z^{\circ}}$-ideals in $C(X)$, Acta. Math. Sin. (Engl. ser), 23 (2007), pp. 989-996.

[5] T. Dube, Contracting the Socle in Rings of Continuous Functions, Rend. Semin. Mat. Univ. Padova, 123 (2010), pp. 37-53.

[6] R. Engelking, General topology (Berlin: Heldermann, 1989).

[7] A. A. Estaji - O. A. S. Karamzadeh, On $C(X)$ Modulo its socle, Comm. Algebra, 31 (2003), pp. 1561-1571.

[8] M. Ghadermazi - M. Namdari, On a-scattered spaces, Far East J. Math. Sci. (FJMS), 32 (2) (2009), pp. 267-274.

[9] L. Gillman - M. JERISON, Rings of continuous functions (Springer, 1976).

[10] K. R. GoodearL, Von Neumann Regular Rings (Pitman, 1979). 
[11] M. HenRIKsen, Topology related to rings of real-valued continuous functions. Where it has been and where it might be going, Recent Progress In General Topology II, eds M. Husek and J. Van Mill (Elsevier Science, 2002), pp. 553-556.

[12] O. A. S. Karamzadeh, On a question of Matlis, Comm. Algebra, 25 (1997), pp. 2717-2726.

[13] O. A. S. KARAMZADEH, Modules whose countably generated submodules are epimorphic image, Colloq. Math. 2 (1982), pp. 7-10.

[14] O. A. S. KaRAMZADEH - A. A. KoOCHAKPOUR, On $\aleph_{0}$-selfinjectivity of strongly regular rings, Comm. Algebra, 27 (1999), pp. 1501-1513.

[15] O. A. S. Karamzadeh - M. Motamedi - S. M. Shahrtash, On rings with a unique proper essential right ideal, Fund. Math. 183 (2004), pp. 229-244.

[16] O. A. S. Karamzadeh - M. Rostami, On the intrinsic topology and some related ideals of $C(X)$, Proc. Amer. Math. Soc. 93 (1985), pp. 179-184.

[17] S. LARSON, A characterization of $f$-rings in which the sum of semiprime lideals is semiprime and its consequences, Comm. Algebra, (1995), pp. 54616481.

[18] R. Levy - M. Matveev, Functional separability, Comment. Math. Univ. Carolin. 51 (2010), pp. 705-711.

[19] R. LeVy - M. D. RICE, Normal P-spaces and the $G_{\delta}$-topology, Colloq. Math. 47 (1981), pp. 227-240.

[20] M. A. Mulero, Algebraic properties of rings of continuous functions, Fund. Math. 149 (1996), pp. 55-66.

[21] A. Pelczynski - Z. Semadeni, Spaces of continuous functions (III), Studia Mathematica, 18 (1959), pp. 211-222.

[22] R. RAPHAEL - R. G. WoODS, On essential ring embeddings and the epimorphic hull of $C(X)$, Theory and Applications of Categories, 14 (2005), pp. 46-52.

[23] W. Rudin, Continuous functions on compact spaces without perfect subsets, Proc. Amer. Math. Soc. 8 (1957), pp. 39-42.

[24] D. RudD, On two sum theorems for ideals of C(X), Michigan Math. J. 17 (1970), pp. 139-141.

[25] A. W. WiCKSTEAD, An intrinsic Characterization of selfinjective semi-prime commutative rings, Proc. R. Ir. Acad. 90 (A) (1989), pp. 117-124.

Manoscritto pervenuto in redazione il 16 Settembre 2011. 
